\title{
ALGEBRAS ASSOCIATED TO THE YOUNG-FIBONACCI LATTICE
}

\author{
SOICHI OKADA
}

\begin{abstract}
The algebra $\mathscr{F}_{n}$ generated by $E_{1}, \ldots, E_{n-1}$ subject to the defining relations $E_{i}^{2}=x_{i} E_{i}(i=1, \ldots, n-1), E_{i+1} E_{i} E_{i+1}=y_{i} E_{i+1} \quad(i=$ $1, \ldots, n-2), E_{i} E_{j}=E_{j} E_{i}(|i-j| \geq 2)$ is shown to be a semisimple algebra of dimension $n$ ! if the parameters $x_{1}, \ldots, x_{n-1}, y_{1}, \ldots, y_{n-2}$ are generic. We also prove that the Bratteli diagram of the tower $\left(\mathscr{F}_{n}\right)_{n \geq 0}$ of these algebras is the Hasse diagram of the Young-Fibonacci lattice, which is an interesting example, as well as Young's lattice, of a differential poset introduced by R. Stanley. A Young-Fibonacci analogue of the ring of symmetric functions is given and studied.
\end{abstract}

\section{INTRODUCTION}

In [S1], R. Stanley introduced a class of partially ordered sets called differential posets, whose prototypical example is Young's lattice $\mathbb{Y}$. S. Fomin [F1] independently defined essentially the same class of graphs, called Y-graphs. (See [F2], [S2] for generalization.) Many enumerative results, concerning the counting of chains or Hasse walks in differential posets or Y-graphs, can be derived by using an algebraic approach (see [S1]) and also by applying a combinatorial method such as Robinson-Schensted-type correspondences (see [F1], [F3], [R1], [R2]). In the case of Young's lattice, these properties reflect the representation theory of the symmetric groups and the theory of symmetric functions.

Fomin [F1] and Stanley [S1] also gave another example of a differential poset, $\mathbb{Y F}$, called the Young-Fibonacci lattice. (In [S1] this lattice is denoted by $Z(1)$.) And Stanley posed a problem [S1, 66 , Problem 8] to give a natural and combinatorial definition of the tower $\left(\mathscr{F}_{n}\right)_{n \geq 0}$ of semisimple algebras, which play the same role to the Young-Fibonacci lattice $\mathbb{Y F}$ as the group algebras of the symmetric groups play to Young's lattice $\mathbb{Y}$. This work is motivated to this problem and the first aim of this article is to give a presentation of $\mathscr{F}_{n}$, which corresponds to that of the symmetric group with respect to the adjacent transpositions. The second aim is to define and study a $\mathbb{Y} F$-analogue of the ring of symmetric functions.

Let us explain in more detail. Young's lattice $\mathbb{Y}$ is the set of all partitions ordered by inclusion of Young (or Ferrers) diagrams. It is well known that the irreducible representations of the symmetric group $\mathfrak{S}_{n}$ are parametrized by $\mathbb{Y}_{n}$, the set of partitions of $n$. If we denote by $V_{\mathfrak{S}_{n}}^{\lambda}$ the irreducible $\mathfrak{S}_{n}$-module

Received by the editors November 10, 1993.

1991 Mathematics Subject Classification. Primary 05E99.

Key words and phrases. Young-Fibonacci lattice, differential poset, Bratteli diagram. 
corresponding to a partition $\lambda$, then the restriction of $V^{\lambda}$ to $\mathfrak{S}_{n-1}$ decomposes as follows:

$$
V_{\mathfrak{S}_{n}}^{\lambda} \downarrow_{\mathfrak{S}_{n-1}} \cong \bigoplus_{\mu} V_{\mathfrak{S}_{n-1}}^{\mu},
$$

where $\mu$ runs over all partitions whose Young diagrams are obtained from that of $\lambda$ by deleting one box. Moreover, the direct sum $R(\mathfrak{S})=\bigoplus_{n \geq 0} R\left(\mathfrak{S}_{n}\right)$ of the character ring $R\left(\mathfrak{S}_{n}\right)$ of $\mathfrak{S}_{n}$ has a structure of graded algebra and there is an algebra isomorphism from $R(\mathfrak{S})$ to the ring $\Lambda$ of symmetric functions. Under this isomorphism, the irreducible character $\chi^{\lambda}$ of $V_{\mathfrak{S}_{n}}^{\lambda}$ corresponds to the Schur function $s_{\lambda}$.

The Young-Fibonacci lattice $\mathbb{Y} F$ is a differential poset consisting of all words with alphabets $\{1,2\}$. (See Section 1 for the definition of the partial order on $\mathbb{Y F}$.) Let $\mathscr{F}_{n}$ be the associative algebra (over a field $K_{0}$ of characteristic 0 ) defined by the following presentation:

$$
\begin{aligned}
\text { generators : } & E_{1}, \ldots, E_{n-1} \\
\text { relations : } & E_{i}^{2}=x_{i} E_{i} \quad(i=1, \ldots, n-1), \\
& E_{i+1} E_{i} E_{i+1}=y_{i} E_{i+1} \quad(i=1, \ldots, n-2), \\
& E_{i} E_{j}=E_{j} E_{i} \quad(\text { if }|i-j| \geq 2) .
\end{aligned}
$$

Suppose that the parameters $x_{1}, \ldots, x_{n-1}, y_{1}, \ldots, y_{n-2} \in K_{0}$ are generic. In Section 2, we will construct irreducible representations of this algebra $\mathscr{F}_{n}$ and prove that $\mathscr{F}_{n}$ is semisimple of dimension $n$ ! and its irreducible representations are indexed by $\mathbb{Y F}_{n}$, the set of elements of $\mathbb{Y F}$ with rank $n$. If we denote by $V_{v}$ the irreducible $\mathscr{F}_{n}$-module corresponding to $v \in \mathbb{Y F}_{n}$, then the branching rule for the restriction to the subalgebra $\mathscr{F}_{n-1}=\left\langle E_{1}, \ldots, E_{n-2}\right\rangle$ is described in the same way as in the case of $\mathbb{Y}$ :

$$
V_{v} \downarrow \mathscr{I}_{n-1} \cong \bigoplus_{w} V_{w}
$$

where $w$ runs over all words covered by $v$ in $\mathbb{Y F}$. In Section 3, we define a graded algebra $R=\bigoplus_{n \geq 0} R_{n}$, whose homogeneous components $R_{n}$ are the free $\mathbb{Z}$-modules with basis corresponding to (the isomorphism classes of) the irreducible representations of $\mathscr{F}_{n}$. This algebra can be considered as a $\mathbb{Y F}$ analogue of the ring $\Lambda$ of symmetric functions. We introduce various basis of $R$, which correspond to Schur functions, complete symmetric functions, and power sum symmetric functions, and study the transition matrices between these basis in Sections 4 and 5. A generalization to the $r$-Young-Fibonacci lattice will be given in Section 6.

\section{Young-FibonacCi LATTICE}

In this section, we collect some notations and properties concerning with the Young-Fibonacci lattice, which will be used in the rest of this paper. The reader is referred to [S1] for the general theory of differential posets and [S1, §5], [S3] for further information of the Young-Fibonacci lattice.

Let $r$ be a positive integer. Let $\mathbb{Y F}^{(r)}$ be the set of all finite words (including the empty word $\varnothing$ ) with alphabets $\left\{1_{0}, \ldots, 1_{r-1}, 2\right\}$. For such a word $v=$ $a_{1} \ldots a_{k} \in \mathbb{Y F}^{(r)}$, we define its rank $|v|=\left|a_{1}\right|+\cdots+\left|a_{k}\right|$, where $\left|1_{m}\right|=1$. And we put $\mathbb{Y F}_{n}^{(r)}=\left\{v \in \mathbb{Y F}^{(r)}:|v|=n\right\}$. 
We define a partial order on $\mathbb{Y F}^{(r)}$ by requiring the following conditions: $\varnothing$ is the minimum element,

$$
\begin{aligned}
& C^{-}\left(1_{m} v\right)=\{v\}, \\
& C^{-}(2 v)=C^{+}(v),
\end{aligned}
$$

where $C^{-}(x)$ (resp. $C^{+}(x)$ ) denotes the set of all elements covered by (resp. covering) $x$. The notation $x \triangleright y$ will be used to mean that $x$ covers $y$. From (1.2) and (1.3), we have

$$
C^{+}(v)=\left\{1_{m} v: m=0, \ldots, r-1\right\} \cup\left\{2 w: w \in C^{-}(v)\right\} .
$$

This poset $\mathbb{Y F}^{(r)}$ is is shown to be a graded lattice, and its rank generating function is given by

$$
\sum_{n \geq 0} \# \mathbb{Y} \mathbb{F}_{n}^{(r)} q^{n}=\left(1-r q-q^{2}\right)^{-1}
$$

In particular, $\# \mathbb{Y} \mathbb{F}_{n}^{(1)}$ is the $n$th Fibonacci number $F_{n}$. We call $\mathbb{Y} \mathbb{F}^{(r)}$ the $r$-Young-Fibonacci lattice.

Let $R_{n}^{(r)}$ be the free $\mathbb{Z}$-module with basis $\left\{s_{v}: v \in \mathbb{Y F}_{n}^{(r)}\right\}$. Put

$$
R^{(r)}=\bigoplus_{n \geq 0} R_{n}^{(r)}
$$

and define a scalar product on $R$ by $\left\langle s_{v}, s_{w}\right\rangle=\delta_{v w}$ for all $v, w \in \mathbb{Y F}^{(r)}$. We introduce two linear maps $U, D: R^{(r)} \rightarrow R^{(r)}$ by putting

$$
U s_{v}=\sum_{w \triangleright v} s_{w}, \quad D s_{v}=\sum_{w \triangleleft v} s_{w} .
$$

In Sections 3 and 6, we will define a structure of graded algebra on $R^{(r)}$.

Proposition $1.1[\mathrm{~S} 1, \S 5]$. The poset $\mathbb{Y F}^{(r)}$ is an r-differential poset. Hence we have $D U-U D=r \mathrm{Id}$, where Id denotes the identity map on $R^{(r)}$.

For $v \in \mathbb{Y} \mathbb{F}_{n}^{(r)}$, let $\Omega^{v}$ be the set of all sequences $\left(v^{(0)}, \ldots, v^{(n)}\right)$ such that $v^{(0)}=\varnothing, v^{(n)}=v$, and $v^{(i)}$ covers $v^{(i-1)}$ for all $i$; that is, $\Omega^{v}$ is the set of all saturated chains from $\varnothing$ to $v$. We denote the cardinality of $\Omega^{v}$ by $e(v)$. From the general theory of differential posets, we have the following proposition:

Proposition 1.2 [S1, Corollary 3.9]. For the $r$-Young-Fibonacci lattice $\mathbb{Y} \mathbb{F}^{(r)}$, one has

$$
\sum_{v \in \mathbb{Y F}_{n}^{(r)}} e(v)^{2}=r^{n} n !
$$

If $r=1$, then we omit the superscript $(r)$, so that we write $\mathbb{Y F}=\mathbb{Y} \mathbb{F}^{(1)}$, $\mathbb{Y F}_{n}=\mathbb{Y F}_{n}^{(1)}, R=R^{(1)}$, and $R_{n}=R_{n}^{(1)}$.

It is convenient to write $v \in \mathbb{Y} \mathbb{F}$ of the form $1^{m_{1}} 21^{m_{2}} 2 \ldots 1^{m_{r}} 21^{m_{r+1}}$, where $r$ is the number of 2 's appearing in $v$ and $m_{i} \geq 0$. The number $m_{1}$ is denoted by $m(v)$ and it will play a role in Section 5 . 


\section{AlgEBRA $\mathscr{F}_{n}$ AND ITS REPRESENTATIONS}

Let $K_{0}$ be a field of characteristic 0 . We work over the base field $K=$ $K_{0}\left(x_{1}, \ldots, y_{1}, \ldots\right)$, the rational function field with indeterminates $x_{1}, \ldots$, $y_{1}, \ldots$

Definition. Let $\mathscr{F}_{n}=\mathscr{F}_{n}\left(x_{1}, \ldots, x_{n-1} ; y_{1}, \ldots, y_{n-2}\right)$ be the associative $K$ algebra with identity 1 defined by the following presentation:

$$
\begin{aligned}
\text { generators : } & E_{1}, \ldots, E_{n-1} \\
\text { relations : } & E_{i}^{2}=x_{i} E_{i} \quad(i=1, \ldots, n-1), \\
& E_{i} E_{j}=E_{j} E_{i} \quad(\text { if }|i-j| \geq 2), \\
& E_{i+1} E_{i} E_{i+1}=y_{i} E_{i+1} \quad(i=1, \ldots, n-2) .
\end{aligned}
$$

In this section, we will construct irreducible representations of $\mathscr{F}_{n}$ by using paths in $\mathbb{Y F}$ (as in [GHJ, Chapter 2], [KM], [W]) and prove that $\mathscr{F}_{n}$ is a semisimple algebra of dimension $n !$.

First we show that the monomials in $E_{1}, \ldots, E_{n-1}$ span the algebra $\mathscr{F}_{n}$.

Lemma 2.1. We define a sequence of subsets $\mathscr{B}_{k}(k=0,1, \ldots, n)$ as follows:

$$
\begin{gathered}
\mathscr{B}_{0}=\mathscr{B}_{1}=\{1\}, \\
\mathscr{B}_{m}=\left\{b E_{m-1} \ldots E_{k}: b \in \mathscr{B}_{m-1}, k=1, \ldots, m\right\} .
\end{gathered}
$$

Here we understand that $E_{m-1} \ldots E_{k}=1$ if $k=m$. Then $\mathscr{B}_{n}$ spans $\mathscr{F}_{n}$. In particular, $\operatorname{dim}_{K} \mathscr{F}_{n} \leq n$ !.

Proof. Let $\mathscr{F}_{m}^{\prime}$ be the $K$-subspace spanned by $\mathscr{B}_{m}$. We prove by induction on $m$ that $\mathscr{F}_{m}^{\prime}$ is stable under the right multiplication by $E_{l}(l=1, \ldots, m-1)$. We will show that $a=b E_{m-1} \ldots E_{k} E_{l} \in \mathscr{F}_{m}^{\prime}$ for $b \in \mathscr{F}_{m-1}^{\prime}, k=1, \ldots, m$, and $l=1, \ldots, m-1$. If $l \leq k-2$, then we have $a=b E_{l} E_{m-1} \ldots E_{k}$ by (2.3). Since $b E_{l} \in \mathscr{F}_{m-1}^{\prime}$ by the induction hypothesis, we have $a \in \mathscr{F}_{m}^{\prime}$. If $l=k-1$, then it is clear that $a \in \mathscr{F}_{m}^{\prime}$. If $l=k$, then by (2.1), we have $a=x_{k} b E_{m-1} \ldots E_{k} \in I_{m}$. If $l>k$, then by using (2.2) and (2.3), we have

$$
\begin{aligned}
a & =b E_{m-1} \ldots E_{l} E_{l-1} E_{l} E_{l-2} \ldots E_{k} \\
& =y_{l-1} b E_{m-1} \ldots E_{l} E_{l-2} \ldots E_{k} \\
& =y_{l-1} b E_{l-2} \ldots E_{k} E_{m-1} \ldots E_{l} .
\end{aligned}
$$

It follows from the induction hypothesis that $a \in \mathscr{F}_{m}^{\prime}$.

In order to describe matrix representations of $\mathscr{F}_{n}$, we associate $\alpha(v) \epsilon$ $K$ to each element $v \in \mathbb{Y F}$. Let $\left(P_{l}\right)_{l \geq 0}$ be the sequence of polynomials $P_{l}\left(x_{1}, \ldots, x_{l} ; y_{1}, \ldots, y_{l-1}\right)$ given by the following recurrence:

$$
P_{0}=1, \quad P_{1}=x_{1}, \quad P_{l}=x_{l} P_{l-1}-y_{l-1} P_{l-2} .
$$

Then $\alpha(v)$ is defined as follows:

$$
\begin{gathered}
\alpha\left(1^{l}\right)=P_{l}\left(x_{1}, \ldots, x_{l} ; y_{1}, \ldots, y_{l-1}\right), \\
\alpha\left(1^{l} 2\right)=P_{l+1}\left(y_{1}, x_{3}, \ldots, x_{l+2} ; x_{1} y_{2}, y_{3}, \ldots, y_{l+1}\right) .
\end{gathered}
$$

In general, if $v$ is of the form $1^{l} 2 u(|u|=m)$, then we put

$$
\alpha\left(1^{l} 2 u\right)=\alpha\left(1^{l} 2\right)\left[x_{j} \rightarrow x_{m+j}, y_{j} \rightarrow y_{m+j}\right] \alpha(u),
$$

where $P[z \rightarrow w]$ indicates that we substitute $w$ for $z$ in $P$. 
Lemma 2.2. For $v \in \mathbb{Y F}_{n}$, we have

$$
\begin{gathered}
\sum_{u \triangleright v} \alpha(u)=x_{n+1} \alpha(v), \\
\alpha(2 v)=y_{n+1} \alpha(v) .
\end{gathered}
$$

Moreover, we have

$$
\sum_{v \in \mathbb{Y F}_{n}} e(v) \alpha(v)=x_{1} \ldots x_{n},
$$

where $e(v)$ is the number of saturated chains from $\varnothing$ to $v$ in $\mathbb{Y F}$.

Proof. The relation (2.9) is clear from the definition (2.7) and $\alpha(2)=y_{1}$. We prove (2.8) by induction on $|v|$. First we consider the case where $v=2 w$. Since $C^{+}(2 w)=\{12 w\} \cup\{2 z: z \triangleright w\}$ by (1.4), we have

$$
\sum_{u \triangleright 2 w} \alpha(u)=\alpha(12)\left[x_{j} \rightarrow x_{m+j}, y_{j} \rightarrow y_{m+j}\right] \alpha(w)+\sum_{z \triangleright w} y_{m+2} \alpha(w),
$$

where $|w|=m$. By using $\alpha(12)=x_{3} y_{1}-x_{1} y_{2}$ and the induction hypothesis, we get

$$
\sum_{u \triangleright 2 w} \alpha(u)=\left(x_{m+3} y_{m+1}-x_{m+1} y_{m+2}\right) \alpha(w)+y_{m+2} x_{m+1} \alpha(w)=x_{m+3} \alpha(2 w) .
$$

If $v=1^{k} 2 w$ for some $k>0$, then

$$
\begin{aligned}
\sum_{u \triangleright 1^{k} 2 w} \alpha(u)= & \alpha\left(1^{k+1} 2\right)\left[x_{j} \rightarrow x_{m+j}, y_{j} \rightarrow y_{m+j}\right] \alpha(w) \\
& +y_{m+k+2} \alpha\left(1^{k-1} 2\right)\left[x_{j} \rightarrow x_{m+j}, y_{j} \rightarrow y_{m+j}\right] \alpha(w),
\end{aligned}
$$

where $|w|=m$. Hence it is enough to show

$$
\alpha\left(1^{k+1} 2\right)+y_{k+2} \alpha\left(1^{k-1} 2\right)=x_{k+3} \alpha\left(1^{k} 2\right) .
$$

But this is clear from the definition (2.5) and (2.6). Similarly we can check the case where $v=1^{n}$.

The remaining equation (2.10) follows from (2.8).

It follows from the definition of differential posets (see Proposition 1.1) and (1.3) that the $e(v)$ 's are uniquely determined by the same recurrence relations as (2.8) with $x_{i}=i$ and $y_{i}=i$, and the initial condition $e(\varnothing)=1$. So we have $\alpha(v)\left[x_{i} \rightarrow i, y_{i} \rightarrow i\right]=e(v)$. In particular, $\alpha(v)$ is a nonzero polynomial.

For $v \in \mathbb{Y F}_{n}$, let $V_{v}$ be the $K$-vector space with basis $\Omega^{v}$. Then $\operatorname{dim} V_{v}=$ $e(v)$. Now define an action $\pi_{v}\left(E_{i}\right)$ of each generator $E_{i}$ on the vector space $V_{v}$ as follows:

$$
\begin{aligned}
& \pi_{v}\left(E_{i}\right)\left(v^{(0)}, \ldots, v^{(i-1)}, v^{(i)}, v^{(i+1)}, \ldots, v^{(n)}\right) \\
& =\left\{\begin{array}{l}
\sum_{z \triangleright v^{(i-1)}} \frac{\alpha(z)}{\alpha\left(v^{(i-1)}\right)}\left(v^{(0)}, \ldots, v^{(i-1)}, z, v^{(i+1)}, \ldots, v^{(n)}\right) \text { if } v^{(i+1)}=2 v^{(i-1)} \\
0 \text { otherwise. }
\end{array}\right.
\end{aligned}
$$


Lemma 2.3. The endomorphisms $\pi_{v}\left(E_{i}\right)$ satisfy the defining relations (2.1)-(2.3) of $\mathscr{F}_{n}$. Hence we obtain a representation $\pi_{v}$ of $\mathscr{F}_{n}$ on $V_{v}$.

Proof. The relation (2.2) is clear from the definition.

Let $T=\left(v^{(0)}, \ldots, v^{(n)}\right) \in \Omega^{v}$. We will check that $\pi_{v}\left(E_{i}\right)^{2} T=x_{i} \pi_{v}\left(E_{i}\right) T$. If $v^{(i+1)} \neq 2 v^{(i-1)}$, then both sides are 0 . If $v^{(i+1)}=2 v^{(i-1)}$, then

$$
\begin{aligned}
\pi_{v}\left(E_{i}\right)^{2} T & =\sum_{w \triangleright v^{(i-1)}} \frac{\alpha(w)}{\alpha\left(v^{(i-1)}\right)} \pi_{v}\left(E_{i}\right) T_{w} \\
& =\sum_{u \triangleright v^{(i-1)}} \sum_{w \triangleright v^{(i-1)}} \frac{\alpha(w)}{\alpha\left(v^{(i-1)}\right)} \frac{\alpha(u)}{\alpha\left(v^{(i-1)}\right)} T_{u} \\
& =\sum_{w \triangleright v^{(i-1)}} \frac{\alpha(w)}{\alpha\left(v^{(i-1)}\right)} \pi_{v}\left(E_{i}\right) T \\
& =x_{i} \pi_{v}\left(E_{i}\right) T \quad(\text { by }(2.8))
\end{aligned}
$$

where $T_{w}=\left(v^{(0)}, \ldots, v^{(i-1)}, w, v^{(i+1)}, \ldots, v^{(n)}\right)$.

Next we check that $\pi_{v}\left(E_{i+1}\right) \pi_{v}\left(E_{i}\right) \pi_{v}\left(E_{i+1}\right) T=y_{i} \pi_{v}\left(E_{i+1}\right) T$. If $v^{(i+2)} \neq$ $2 v^{(i)}$, then both sides are 0 . If $v^{(i+2)}=2 v^{(i)}$, then

$$
\begin{aligned}
\pi_{v}( & \left.E_{i+1}\right) \pi_{v}\left(E_{i}\right) \pi_{v}\left(E_{i+1}\right) T=\sum_{w \triangleright v^{(i)}} \frac{\alpha(w)}{\alpha\left(v^{(i)}\right)} \pi_{v}\left(E_{i+1}\right) \pi_{v}\left(E_{i}\right) T_{v^{(i)}, w} \\
= & \frac{\alpha\left(2 v^{(i-1)}\right)}{\alpha\left(v^{(i)}\right)} \pi_{v}\left(E_{i+1}\right) \pi_{v}\left(E_{i}\right) T_{v^{(i)}, 2 v^{(i-1)}} \\
= & \frac{\alpha\left(2 v^{(i-1)}\right)}{\alpha\left(v^{(i)}\right)} \sum_{u \triangleright v^{(i-1)}} \frac{\alpha(u)}{\alpha\left(v^{(i-1)}\right)} \pi_{v}\left(E_{i+1}\right) T_{u, 2 v^{(i-1)}} \\
= & \frac{\alpha\left(2 v^{(i-1)}\right)}{\alpha\left(v^{(i)}\right)} \frac{\alpha\left(v^{(i)}\right)}{\alpha\left(v^{(i-1)}\right)} \pi_{v}\left(E_{i+1}\right) T \\
= & y_{i} \pi_{v}\left(E_{i+1}\right) T \quad(\text { by }(2.9))
\end{aligned}
$$

where $T_{u, w}=\left(v^{(0)}, \ldots, v^{(i-1)}, u, w, v^{(i+2)}, \ldots, v^{(n)}\right)$.

If $v$ covers $w$, then $V_{w}$ can be considered as a subspace of $V_{v}$ by identifying $\left(v^{(0)}, \ldots, v^{(n-2)}, w\right) \in \Omega^{w}$ with $\left(v^{(0)}, \ldots, v^{(n-2)}, w, v\right) \in \Omega^{v}$.

Lemma 2.4. If we restrict the representation $\pi_{v}$ to the subalgebra $\mathscr{F}_{n-1}$ generated by $E_{1}, \ldots, E_{n-2}$, then $V_{v}$ decomposes as follows:

$$
V_{v} \downarrow \mathscr{F}_{n-1} \cong \bigoplus_{w \triangleleft v} V_{w}
$$

Proof. This is clear from the definition (2.11) of the action of $E_{1}, \ldots ; E_{n-2}$.

Lemma 2.5. The representations $\left(\pi_{v}, V_{v}\right)$ of $\mathscr{F}_{n}$ are irreducible and pairwise inequivalent.

Proof. We proceed by induction on $n$. First we show the irreducibility of $\left(\pi_{v}, V_{v}\right)$. Let $W \neq\{0\}$ be an $\mathscr{F}_{n}$-submodule of $V_{v}$.

If $v=1 v^{\prime}$, then Lemma 2.4 implies that $V_{1 v^{\prime}}=V_{v^{\prime}}$ as on $\mathscr{F}_{n-1}$-module. By the induction hypothesis, it is irreducible over $\mathscr{F}_{n-1}$. Hence we have $W=$ $V_{v^{\prime}}=V_{1 v^{\prime}}$. 
If $v=2 v^{\prime \prime}$, then there exists an element $x$ such that $x$ covers $v^{\prime \prime}$ and $V_{x} \subset W$, because the irreducible decomposition of $V_{v} \downarrow \mathscr{F}_{n-1}$ is multiplicityfree. Now let $y \neq x$ be an element covering $v^{\prime \prime}$ and consider two chains $T=\left(v^{(0)}, \ldots, v^{(n-3)}, v^{\prime \prime}, x, v\right)$ and $T^{\prime}=\left(v^{(0)}, \ldots, v^{(n-3)}, v^{\prime \prime}, y, v\right) \in \Omega^{v}$. Let $z_{y}$ be the minimal central idempotent of $\mathscr{F}_{n-1}$ corresponding to $\pi_{y}$. Then it follows from the definition of $\pi_{v}\left(E_{n-1}\right)$ that

$$
\pi_{v}\left(z_{y}\right) \pi_{v}\left(E_{n-1}\right) T=\frac{\alpha(y)}{\alpha\left(v^{\prime \prime}\right)} T^{\prime} \in W .
$$

Hence we have $W \cap V_{y} \neq\{0\}$. Since $V_{y}$ is an irreducible $\mathscr{F}_{n-1}$-module by the induction hypothesis, we see that $V_{y} \subset W$. Recalling that $y$ is arbitrary, we have $W=V_{x} \oplus \bigoplus_{y \triangleright v^{\prime \prime}, y \neq x} V_{y}=V_{v}$.

Next we show that the $\left(\pi_{v}, V_{v}\right)$ are inequivalent. Suppose that $V_{v} \cong V_{w}$ as as $\mathscr{F}_{n}$-module. Then, by Lemma 2.4 , we have $C^{-}(v)=C^{-}(w)$. Except for the case where $v=11$ and $w=2$, it follows from definition (1.2) and (1.3) that $v=w$. In the exceptional case, it follows from $\pi_{11}\left(E_{1}\right)=0$ and $\pi_{2}\left(E_{1}\right)=x_{1}$ Id that $V_{11} \not V_{2}$.

Now we are in position to prove the main theorem.

Theorem 2.6. (1) The algebra $\mathscr{F}_{n}$ is semisimple.

(2) The set $\mathscr{B}_{n}$ of monomials defined by (2.4) is a basis of $\mathscr{F}_{n}$. In particular, $\operatorname{dim} \mathscr{F}_{n}=n !$.

(3) The $V_{v}$ 's $\left(v \in \mathbb{Y F}_{n}\right)$ give a complete set of irreducible $\mathscr{F}_{n}$-modules.

Proof. Let $\operatorname{rad} \mathscr{F}_{n}$ be the radical of $\mathscr{F}_{n}$. Then, by Lemma 2.5, we have

$$
\begin{aligned}
\operatorname{dim}\left(\mathscr{F}_{n} / \operatorname{rad} \mathscr{F}_{n}\right) & \geq \operatorname{dim}\left(\bigoplus_{v \in \mathbb{Y F}_{n}} \pi_{v}\left(\mathscr{F}_{n}\right)\right) \geq \sum_{v \in \mathbb{Y F}_{n}}\left(\operatorname{dim} V_{v}\right)^{2} \\
& =\sum_{v \in \mathbb{Y} \mathbb{F}_{n}} e(v)^{2}=n ! .
\end{aligned}
$$

Here we have used Proposition 1.2. On the other hand, Lemma 2.1 implies that $\operatorname{dim} \mathscr{F}_{n} \leq n !$. Therefore we obtain the desired results.

For $a \in \mathscr{F}_{n}$, we define

$$
\operatorname{Tr}^{(n)}(a)=\left(x_{1} \ldots x_{n}\right)^{-1} \sum_{v \in \mathbb{Y F}_{n}} \alpha(v) \operatorname{tr}_{v_{v}}\left(\pi_{v}(a)\right),
$$

where $\operatorname{tr}_{V_{v}}$ denotes the usual trace on the vector space $V_{v}$. Then $\operatorname{Tr}^{(n)}$ has the following properties similar to those of the Markov trace on the Iwahori-Hecke algebra of the symmetric group (see [W, §3]).

Proposition 2.7. The functional $\operatorname{Tr}^{(n)}$ defined by (2.12) satisfies the following.

(1) $\operatorname{Tr}^{(n)}(1)=1$.

(2) $\operatorname{Tr}^{(n)}(a b)=\operatorname{Tr}^{(n)}(b a)$.

(3) If $a \in \mathscr{F}_{n-1}$, then $\operatorname{Tr}^{(n)}\left(a E_{n-1}\right)=y_{n-1} \operatorname{Tr}^{(n)}(a)$.

(4) If $a \in \mathscr{F}_{n-1}$, then $\operatorname{Tr}^{(n)}(a)=\operatorname{Tr}^{(n-1)}(a)$. 
Proof. (1) follows from (2.10). (2) is clear from definition (2.12).

(3) Given $T \in \Omega^{v}$, let $p_{T}$ be the minimal idempotent of $\mathscr{F}_{n-1}$ such that

$$
\pi_{w}\left(p_{T}\right)= \begin{cases}E_{T T} & (w=v), \\ 0 & (w \neq v),\end{cases}
$$

where $E_{T T}$ denotes the matrix unit, i.e., the linear map defined by $E_{T T}(S)=$ $\delta_{S, T} T$ for $S \in \Omega^{v}$. Since $\sum_{v \in \mathbb{Y F}_{n-1}} \sum_{T \in \Omega^{v}} p_{T}=1$, it is enough to show

$$
\operatorname{Tr}^{(n)}\left(a E_{n-1} p_{T}\right)=y_{n-1} \operatorname{Tr}^{(n)}\left(a p_{T}\right) \text {. }
$$

Since $p_{T}$ is a minimal idempotent, there exists a scalar $\gamma(a) \in K$ such that $p_{T} a p_{T}=\gamma(a) p_{T}$. Hence we have

$$
\operatorname{Tr}^{(n)}\left(a p_{T}\right)=\left(x_{1} \ldots x_{n}\right)^{-1} \gamma(a) \alpha(v) .
$$

On the other hand, if $z_{v}$ is the minimal central idempotent of $\mathscr{F}_{n}$ corresponding to $\pi_{v}$, then we have

$$
z_{v} E_{n-1} p_{T}=\frac{\alpha(v)}{\alpha\left(v^{(n-2)}\right)} p_{T} p_{\widetilde{T}}
$$

where $T=\left(v^{(0)}, \ldots, v^{(n-2)}, v^{(n-1)}\right), \widetilde{T}=\left(v^{(0)}, \ldots, v^{(n-2)}, v^{(n-1)}, 2 v^{(n-2)}\right)$. Hence we have

$$
\begin{aligned}
\operatorname{Tr}^{(n)}\left(a E_{n-1} p_{T}\right) & =\frac{\alpha(v)}{\alpha\left(v^{(n-2)}\right)} \gamma(a) \operatorname{Tr}^{(n)}\left(p_{\widetilde{T}}\right) \\
& =\left(x_{1} \ldots x_{n}\right)^{-1} \frac{\alpha(v)}{\alpha\left(v^{(n-2)}\right)} \gamma(a) \alpha\left(2 v^{(n-2)}\right) \\
& =\left(x_{1} \ldots x_{n}\right)^{-1} y_{n-1} \alpha(v) \gamma(a) .
\end{aligned}
$$

Hence we obtain (3).

(4) For $a \in \mathscr{F}_{n-1}$, by using (2.8) and Lemma 2.5, we have

$$
\begin{aligned}
\operatorname{Tr}^{(n)}(a) & =\left(x_{1} \ldots x_{n}\right)^{-1} \sum_{|w|=n-1} \sum_{v \triangleright w} \alpha(v) \operatorname{tr}_{V_{w}}\left(\pi_{w}(a)\right) \\
& =\left(x_{1} \ldots x_{n}\right)^{-1} \sum_{|w|=n-1} x_{n} \alpha(w) \operatorname{tr}_{V_{w}}\left(\pi_{w}(a)\right) \\
& =\operatorname{Tr}^{(n-1)}(a) . \quad \square
\end{aligned}
$$

Remark. The proof of this section is on the same line as [KM] and [W].

Finally we mention the specialization of the parameters $x_{1}, \ldots, x_{n-1}, y_{1}$, $\ldots, y_{n-2}$. The above argument guarantees the following theorem.

Theorem 2.8. Let $\xi_{1}, \ldots, \xi_{n-1}, \eta_{1}, \ldots, \eta_{n-2}$ be elements of the field $K_{0}$. Let $\overline{\mathscr{F}}_{n}=\mathscr{F}_{n}\left(\xi_{1}, \ldots, \xi_{n-1} ; \eta_{1}, \ldots, \eta_{n-2}\right)$ be the algebra over $K_{0}$ generated by $E_{1}, \ldots, E_{n-1}$ with their fundamental relations given by (2.1)-(2.3), where the $x_{i}$ 's and $y_{j}$ 's are replaced by $\xi_{i}$ 's and $\eta_{j}$ 's respectively. If

$$
\alpha(v)\left[x_{i} \rightarrow \xi_{i}, y_{j} \rightarrow \eta_{j}\right] \neq 0
$$

for all words $v$ with $|v| \leq n-1$, then $\overline{\mathscr{F}_{n}}$ is a semisimple algebra of dimension $n !$.

Remark. The above argument can be easily generalized to the differential poset $T(N)$, which is obtained from the partial differential poset $\mathbb{Y}_{[N]}=\bigsqcup_{k=0}^{N} \mathbb{Y}_{k}$ by 
iterating Wagner's construction. (See [S1, pp. 957-958].) Let $\mathscr{T}(N)_{n}$ be the associative algebra over the field $K(q)$ with generators $T_{1}, \ldots, T_{N-1}, E_{N}, \ldots$, $E_{n-1}$ and the following defining relations:

$$
\begin{aligned}
& \left(T_{i}-q\right)\left(T_{i}+q^{-1}\right)=0 \quad(i=1, \ldots, N-1), \\
& T_{i} T_{i+1} T_{i}=T_{i+1} T_{i} T_{i+1} \quad(i=1, \ldots, N-2), \\
& T_{i} T_{j}=T_{j} T_{i} \quad(|i-j| \geq 2), \\
& E_{N}^{2}=[l] E_{N}, \\
& E_{N} T_{N-1} E_{N}=q^{l} E_{N}, \\
& E_{i}^{2}=x_{i} E_{i} \quad(i=N+1, \ldots, n-1), \\
& E_{i+1} E_{i} E_{i+1}=y_{i} E_{i+1} \quad(i=N, \ldots, n-2), \\
& E_{i} T_{j}=T_{j} E_{i} \quad(|i-j| \geq 2), \\
& E_{i} E_{j}=E_{j} E_{i} \quad(|i-j| \geq 2),
\end{aligned}
$$

where $[l]=\left(q^{l}-q^{-l}\right) /\left(q-q^{-1}\right)$. Then one can show that the Bratteli diagram of the tower $\left(\mathscr{T}(N)_{n}\right)_{n>0}$ is the Hasse diagram of $T(N)$. Note that the algebras $\mathscr{T}(N)_{n}$ for $n \leq N$ are the Iwahori-Hecke algebra of the symmetric group $\mathfrak{S}_{n}$. And M. Kosuda and J. Murakami [KM] have shown that, if $l \geq N+1$, then the algebra $\mathscr{T}(N)_{N+1}$ is isomorphic to the centralizer algebra of the quantum group $U_{q}(\mathfrak{g l}(l, \mathbb{C}))$ on the space $V^{\otimes N} \otimes V^{*}$, where $V$ is the $l$-dimensional vector representation of $U_{q}(\mathfrak{g l}(l, \mathbb{C}))$.

\section{YF-ANALOGUE OF THE RING OF SYMMETRIC FUNCTIONS}

In this section, we give a definition of a graded algebra structure on $R=$ $\bigoplus_{n \geq 0} R_{n}$, which becomes a $\mathbb{Y} \mathbb{F}$-analogue of the ring $\Lambda$ of symmetric functions. Many of the results in the following sections have counterparts in the theory of symmetric functions. (See [M].)

Let $\mathscr{F}_{m, n}$ be the subalgebra of $\mathscr{F}_{m+n}\left(x_{1}, \ldots, x_{m+n-1} ; y_{1}, \ldots, y_{m+n-2}\right)$ generated by $E_{1}, \ldots, E_{m-1}, E_{m+1}, \ldots, E_{m+n-1}$. Then it follows from Theorem $2.7(2)$ that

$$
\begin{aligned}
\mathscr{F}_{m, n} \cong & \mathscr{F}_{m}\left(x_{1}, \ldots, x_{m-1} ; y_{1}, \ldots, y_{m-2}\right) \\
& \otimes \mathscr{F}_{n}\left(x_{m+1}, \ldots, x_{m+n-1} ; y_{m+1}, \ldots, y_{m+n-2}\right) .
\end{aligned}
$$

So $\mathscr{F}_{m, n}$ is a semisimple algebra. If $|w|=m+n$ and $|u|=m$, then let $\Omega^{w / u}$ be the set of all saturated chains from $u$ to $w$ and $V_{w / u}$ the vector space with basis $\Omega^{w / u}$. Note that $V_{w / u}=\{0\}$ unless $w>u$. We define an action $\pi_{w / u}\left(E_{i}\right)$ on $\left(v^{(0)}, \ldots, v^{(n)}\right) \in \Omega^{w / u}$ by the same formula as (2.11) with $\alpha(z)$ and $\alpha\left(v^{(i-1)}\right)$ replaced by $\alpha(z)\left[x_{j} \rightarrow x_{m+j}, y_{j} \rightarrow y_{m+j}\right]$ and $\alpha\left(v^{(i-1)}\right)\left[x_{j} \rightarrow x_{m+j}, y_{j} \rightarrow y_{m+j}\right]$ respectively. Then this action of generators affords a representation $\pi_{w / u}$ of $\mathscr{F}_{n}\left(x_{m+1}, \ldots, x_{m+n-1} ; y_{m+1}, \ldots, y_{m+n-2}\right)$ on $V_{w / u}$. (See the proof of Lemma 2.3.)

Proposition 3.1. If $|w|=m+n$,

$$
V_{w} \downarrow \mathscr{I}_{m, n} \cong \bigoplus_{|u|=m} V_{u} \otimes V_{w / u}
$$

as $\mathscr{F}_{m, n}$-module. 
Now define a product on $R$ by

$$
s_{u} s_{v}=\sum_{w \in \mathbb{Y F}_{m+n}} c_{u v}^{w} s_{w}
$$

for $u \in \mathbb{Y F}_{m}$ and $v \in \mathbb{Y F}_{n}$, where the structure constant $c_{u v}^{w}$ is defined as follows. Let $V_{u}$ (resp. $\left.V_{v}\right)$ be the irreducible $\mathscr{F}_{m}\left(x_{1}, \ldots, x_{m-1} ; y_{1}, \ldots, y_{m-2}\right)$ module (resp. $\mathscr{F}_{n}\left(x_{m+1}, \ldots, x_{m+n-1} ; y_{m+1}, \ldots, y_{m+n-2}\right)$-module) corresponding to $u \in \mathbb{Y F}_{m}$ (resp. $v \in \mathbb{Y F}_{n}$ ). Then $c_{u v}^{w}$ is defined to be the multiplicity of the irreducible $\mathscr{F}_{m+n}$-module $V_{w}$ in the induced module $\mathscr{F}_{m+n} \otimes_{\mathscr{F}_{m, n}}\left(V_{u} \otimes V_{v}\right)$. By Frobenius reciprocity, we see that $c_{u v}^{w}$ is the multiplicity of the irreducible $\mathscr{F}_{m, n}$-module $V_{u} \otimes V_{v}$ in the restriction $V_{w} \downarrow_{\mathscr{F}_{m, n}}$. This product makes $R$ an associative graded algebra.

Proposition 3.2. Suppose that $w \in \mathbb{Y F}_{m+2}$ and $u \in \mathbb{Y F}_{m}$ satisfy $w>u$. Then, as an $\mathscr{F}_{2}\left(x_{m+1}\right)$-module,

$$
V_{w / u} \cong \begin{cases}\left(V_{11}\right)^{\oplus d-1} \oplus V_{2} & \text { if } w=2 u, \\ V_{11} & \text { otherwise },\end{cases}
$$

where $d=\# C^{+}(u)$.

Proof. This is clear by considering the action of $E_{1}$.

\section{Proposition 3.3.}

(1) $s_{v} s_{1}=\sum_{w \triangleright v} s_{w}$.

(2) $s_{v} s_{2}=s_{2 v}$.

(3) $s_{1 v}=s_{v} s_{1}-\left(\sum_{z \triangleleft v} s_{z}\right) s_{2}$.

Proof. (1) is clear from Lemma 2.4 and Proposition 3.1. (2) follows from Propositions 3.1 and 3.2. (3) is a direct consequence of (1) and (2) because of (1.4).

The abstract structure of $R$ is given by the following theorem.

Theorem 3.4. Let $\mathbb{Z}\langle X, Y\rangle$ be the noncommutative polynomial ring with grading given by $\operatorname{deg} X=1$ and $\operatorname{deg} Y=2$. Then there exists an algebra isomorphism $\varphi: \mathbb{Z}\langle X, Y\rangle \rightarrow R$ such that $\varphi(X)=s_{1}$ and $\varphi(Y)=s_{2}$.

Proof. There exists an algebra homomorphism $\varphi: \mathbb{Z}\langle X, Y\rangle \rightarrow R$ such that $\varphi(X)=s_{1}$ and $\varphi(Y)=s_{2}$. By Proposition 3.3, this homomorphism $\varphi$ is surjective. On the other hand, the homogeneous components of degree $n$ in $\mathbb{Z}\langle X, Y\rangle$ and $R$ are both free $\mathbb{Z}$-modules of rank $F_{n}$ ( $n$th Fibonacci number). Hence $\varphi$ is an isomorphism.

\section{Proposition 3.5 .}

$$
s_{u 2 v}=s_{v} s_{u 2} .
$$

In particular, for $v=1^{m_{1}} 21^{m_{2}} 2 \ldots 21^{m_{r+1}}$,

$$
s_{v}=s_{1^{m_{r+1}}} s_{1^{m_{r}} 2} \ldots s_{1^{m_{1} 2}} .
$$

Proof. We prove (3.1) by induction on $|u|$. If $u=\varnothing$, then (3.1) reduces to Proposition 3.3(2). If $u=2 u^{\prime \prime}$, then by Proposition 3.3(2) and the induction hypothesis,

$$
s_{2 u^{\prime \prime} 2 v}=s_{u^{\prime \prime} 2 v} S_{2}=s_{v} S_{u^{\prime \prime} 2} S_{2}=s_{v} S_{2 u^{\prime \prime} 2}
$$


If $u=1 u^{\prime}$, then it follows from Proposition 3.3(3) and the induction hypothesis that

$$
s_{1 u^{\prime} 2 v}=s_{u^{\prime} 2 v} s_{1}-\left(\sum_{x \triangleleft u^{\prime} 2 v} s_{x}\right) s_{2}, \quad s_{v} s_{1 u^{\prime} 2}=s_{v} s_{u^{\prime} 2} s_{1}-s_{v}\left(\sum_{y \triangleleft u^{\prime} 2} s_{y}\right) s_{2} .
$$

Hence it suffices to show that

$$
\sum_{x \triangleleft w 2 v} s_{x}=\sum_{y \triangleleft w 2} s_{v} s_{y} .
$$

We show (3.2) by induction on $|w|$. The case where $w=\varnothing$ follows from Proposition 3.3(1). If $w=1 w^{\prime}$, then

$$
\sum_{x \triangleleft 1 w^{\prime} 2 v} s_{x}=s_{w^{\prime} 2 v}, \quad \sum_{y \triangleleft 1 w^{\prime} 2} s_{v} s_{y}=s_{v} s_{w^{\prime} 2} .
$$

Here we use the induction hypothesis on $|w|$ to obtain (3.2) for $w=1 w^{\prime}$. If $w=2 w^{\prime \prime}$, then

$$
\sum_{x \triangleleft 2 w^{\prime \prime} 2 v} s_{x}=s_{1 w^{\prime \prime} 2 v}+\sum_{t \triangleleft w^{\prime \prime} 2 v} s_{t} s_{2}, \quad \sum_{y \triangleleft 2 w^{\prime \prime} 2} s_{v} s_{y}=s_{v} s_{1 w^{\prime \prime 2}}+\sum_{z \triangleleft w^{\prime \prime 2}} s_{v} s_{z} s_{2} .
$$

Now from the induction hypothesis on $|u|$ and $|w|$, we have

$$
s_{1 w^{\prime \prime 2} 2 v}=s_{v} s_{1 w^{\prime \prime 2}}, \quad \sum_{t \triangleleft w^{\prime \prime 2} 2 v} s_{t} s_{2}=\sum_{z \triangleleft w^{\prime \prime 2}} s_{v} s_{z} s_{2} .
$$

This completes the proof of (3.2), hence (3.1).

This proposition, together with Proposition 3.3, enables us to express $s_{v}$ as a "determinant" of the matrix having noncommutative entries $s_{1}, s_{2}$ (and 0 , $1)$.

There is an involutive automorphism $\omega$ of the poset $\mathbb{Y F}$ such that

$$
\omega(v 11)=v 2, \quad \omega(v 2)=v 11, \quad \omega(v 21)=v 21 .
$$

Then we can define a linear automorphism $\widetilde{\omega}$ of $R$ by $\widetilde{\omega}\left(s_{v}\right)=s_{\omega(v)}$. However $\widetilde{\omega}$ is not an algebra homomorphism: in fact,

$$
\widetilde{\omega}\left(s_{v} s_{1}\right)=\widetilde{\omega}\left(s_{v}\right) s_{1}, \quad \widetilde{\omega}\left(s_{v} s_{2}\right)=\widetilde{\omega}\left(s_{v}\right) s_{2} \quad(v \neq \varnothing) .
$$

Hence, for $v \neq \varnothing$, we have $\widetilde{\omega}\left(s_{v} s_{w}\right)=\widetilde{\omega}\left(s_{v}\right) s_{w}$.

\section{YF-ANALOGUE OF KOSTKA NUMBERS AND THE LITTLEWOOD-RICHARDSON RULE}

Definition. For $w=b_{1} \ldots b_{l} \in \mathbb{Y F}_{n}$, we define

$$
h_{w}=s_{b_{l}} \ldots s_{b_{1}} \text {. }
$$

Note that the order of product in $h_{w}$ is reversed to that of $w$. For $v, w=$ $b_{1} \ldots b_{l} \in \mathbb{Y F}_{n}$, let $\mathscr{K}_{v w}$ be the set of sequences $\left(v^{(0)}, \ldots, v^{(l)}\right)$ from $v^{(0)}=\varnothing$ to $v^{(l)}=v$ satisfying

(1) If $b_{i}=1$, then $v^{(l-i+1)}$ covers $v^{(l-i)}$.

(2) If $b_{i}=2$, then $v^{(l-i+1)}=2 v^{(l-i)}$.

We put $K_{v w}=\# \mathscr{K}_{v w}$ and call this a $\mathbb{Y} F-$ Kostka number.

By definition, we have $K_{v, 1^{n}}=e(v)$ if $|v|=n$. Then the following proposition is an immediate consequence of Proposition 3.3. 
Proposition 4.1. For $w \in \mathbb{Y}_{n}$, one has

$$
h_{w}=\sum_{v \in \mathbb{Y F}_{n}} K_{v w} s_{v}
$$

This corresponds to the Young's rule for the representation of the symmetric groups (see [JK, 2.8.5]).

Now we introduce a partial order $\succeq$ (called dominance order) on each graded component $\mathbb{Y F}_{n}$ of the Young-Fibonacci lattice. For $v=a_{1} \ldots a_{k}, w=b_{1} \ldots b_{l}$ $\in \mathbb{Y}_{n}$, we define $v \succeq w$ if $a_{1}+\cdots+a_{i} \geq b_{1}+\cdots+b_{i}$ for all $i=1,2, \ldots$, $\min (k, l)$.

Theorem 4.2. The following are equivalent for $v, w \in \mathbb{Y F}_{n}$ :

(1) $v \succeq w$

(2) $K_{v w} \neq 0$.

(3) $K_{u v} \leq K_{u w}$ for all $u \in \mathbb{Y}_{n}$.

Proof. (1) $\Rightarrow$ (3) It is enough to consider the case where either

(a) $v=a_{1} \ldots a_{i} 21 a_{i+3} \ldots a_{k}, w=a_{1} \ldots a_{i} 12 a_{i+3} \ldots a_{k}$, or

(b) $v=a_{1} \ldots a_{i} 2, w=a_{1} \ldots a_{i} 11$.

In case (a), by Proposition 3.3(3),

$$
\begin{aligned}
h_{w}-h_{v} & =s_{a_{k}} \ldots s_{a_{i+3}}\left(s_{2} s_{1}-s_{1} s_{2}\right) s_{a_{i}} \ldots s_{a_{1}} \\
& =s_{a_{k}} \ldots s_{a_{i+3}} s_{12} s_{a_{i}} \ldots s_{a_{1}} .
\end{aligned}
$$

Hence $K_{u w}-K_{u v}$ is nonnegative because it is the multiplicity of $V^{u}$ in the $\mathscr{F}_{n}$-module induced from $V^{a_{k}} \otimes \cdots \otimes V^{a_{i+3}} \otimes V^{12} \otimes V^{a_{i}} \otimes \cdots \otimes V^{a_{1}}$. Case (b) is similarly proved by using $s_{1}^{2}-s_{2}=s_{11}$.

(3) $\Rightarrow$ (2) If we take $u=v$ in (3), we have $K_{v w} \geq K_{v v}=1$.

(2) $\Rightarrow$ (1) We proceed by induction on $n$. Let $v=a_{1} \ldots a_{k}$ and $w=$ $b_{1} \ldots b_{l}$. And fix a sequence $\left(v^{(0)}, \ldots, v^{(l)}\right) \in \mathscr{K}_{v}, w$.

If $a_{1}=b_{1}=1$, then $\left(v^{(0)}, \ldots, v^{(l-1)}\right) \in \mathscr{K}_{v^{\prime}, w^{\prime}}$, where $v^{\prime}=a_{2} \ldots a_{l}$ and $w^{\prime}=b_{2} \ldots b_{k}$. By the induction hypothesis, we have $a_{2}+\cdots+a_{i} \geq b_{2}+\cdots+b_{i}$ for all $i$, Hence we have $v \succeq w$. If $b_{1}=2$, then $v=v^{(l)}=2 v^{(l-1)}$, so that $a_{1}=2$. Then we can conclude $v \succeq w$ in a similar way.

Suppose that $a_{1}=2$ and $b_{1}=1$. Since $v^{(l-1)}$ is covered by $v$, we have either

(a) $v^{(l)}=2^{p} a_{p+1} \ldots a_{l}, v^{(l-1)}=2^{p-1} 1 a_{p+1} \ldots a_{l}$, or

(b) $v^{(l)}=2^{p-1} 1 a_{p+1} \ldots a_{l}, v^{(l-1)}=2^{p-1} a_{p+1} \ldots a_{l}$.

Let $v^{(l-1)}=c_{1} \ldots c_{m}$. In case $(\mathrm{a})$, by the induction hypothesis, we have $c_{1}+$ $\cdots+c_{i} \geq b_{2}+\cdots+b_{i+1}$. Since $a_{j} \geq c_{j}$ for all $j$, we have

$$
\begin{aligned}
a_{1}+\cdots+a_{i} & \geq c_{1}+\cdots+c_{i} \geq b_{2}+\cdots+b_{i}+b_{i+1} \\
& \geq b_{2}+\cdots+b_{i}+1=b_{1}+\cdots+b_{i} .
\end{aligned}
$$

In case (b), by the induction hypothesis, we have $c_{1}+\cdots+c_{i} \geq b_{2}+\cdots+b_{i+1}$. If $i \leq p-1$, then the proof is similar to that of case (a). If $i \geq p$, then we see that

$$
\begin{aligned}
a_{1}+\cdots+a_{i} & =c_{1}+\cdots+c_{p-1}+1+c_{p}+\cdots+c_{i-1} \\
& \geq b_{2}+\cdots+b_{i}+1=b_{1}+\cdots+b_{i} .
\end{aligned}
$$

There are recurrence formulas for the $\mathbb{Y} F-K o s t k a$ numbers $K_{v w}$. 


\section{Proposition 4.3.}

(1) $K_{1 v, 1 w}=K_{v, w}$.

(2) $K_{1 v, 2 w}=0$.

(3) $K_{2 v, 1 w}=\sum_{u \triangleright v} K_{u, w}$.

(4) $K_{2 v, 2 w}=K_{v, w}$.

Proof. Easily follows from the definition.

All matrices considered in the following have rows and columns indexed by $\mathbb{Y F}_{n}$ in dominance order. We put $K_{n}=\left(K_{v}, w\right)_{v, w \in \mathbb{Y F}_{n}}$. For example,

$$
\begin{aligned}
& \begin{array}{llllllll}
221 & 212 & 2111 & 122 & 1211 & 1121 & 1112 & 11111
\end{array}
\end{aligned}
$$

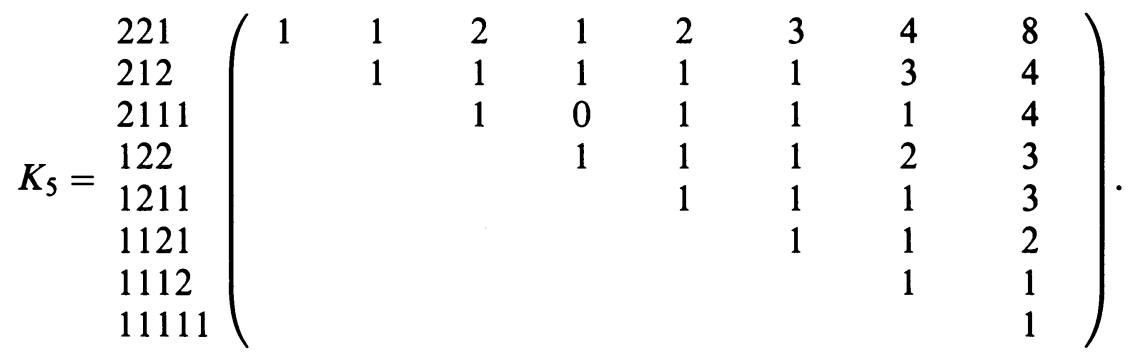

Let $D_{n}=\left(D_{u v}\right)_{u \in \mathrm{YF}_{n-1}, v \in \mathrm{YF}_{n}}$ be the matrix describing the covering relation between $\mathbb{Y} \mathbb{F}_{n}$ and $\mathbb{Y} \mathbb{F}_{n-1}$, so that

$$
D_{u v}= \begin{cases}1 & \text { if } u \triangleleft v \\ 0 & \text { otherwise. }\end{cases}
$$

By definition (1.2) and (1.3), $D_{n+1}$ is of the form

$$
D_{n+1}=\left(\begin{array}{ll}
{ }^{t} D_{n} & I_{F_{n}}
\end{array}\right) \text {, }
$$

where $I_{k}$ is the $k \times k$ identity matrix. Then we can rewrite Proposition 4.3 in matrix form:

$$
K_{n+1}=\left(\begin{array}{cc}
K_{n-1} & D_{n} K_{n} \\
0 & K_{n}
\end{array}\right) .
$$

Remark. Recently T. Halverson and A. Ram [HR] show that the matrix $K_{n}$ appears as the character table of $\mathscr{F}_{n}$. Namely, if we define an element $e_{w} \in \mathscr{F}_{n}$ by $e_{\varnothing}=e_{1}=1$ and

$$
e_{w}= \begin{cases}e_{w^{\prime}} & \text { if } w=1 w^{\prime}, \\ \frac{1}{x_{n-1}} E_{n-1} e_{w^{\prime \prime}} & \text { if } w=2 w^{\prime \prime},\end{cases}
$$

then we have $\operatorname{tr}_{V_{v}}\left(\pi_{v}\left(e_{w}\right)\right)=K_{v w}$.

Definition. Let $u, v, w$ be three elements of $\mathbb{Y F}$ satisfying $|u|+|v|=|w|$, and write $v=a_{1} \ldots a_{k}=1^{m_{1}} 2 \ldots 21^{m_{r+1}}$. Then we define $\mathscr{L}_{w / u, v}$ to be the set of all sequences $\left(w^{(0)}, \ldots, w^{(k)}\right)$ from $u=w^{(0)}$ to $w=w^{(k)}$ satisfying

(1) If $a_{i}=1$, then $w^{(k-i+1)}$ covers $w^{(k-i)}$.

(2) If $a_{i}=2$, then $w^{(k-i+1)}=2 w^{(k-i)}$.

(3) The triple $\left(w^{(j-1)}, w^{(j)}, w^{(j+1)}\right)$ is not of the form $\left(w^{(j-1)}, 1 w^{(j-1)}\right.$, $\left.2 w^{(j-1)}\right)$ for any $j=1, \ldots, m_{r+1}-1$.

(4) If $a_{i}=1$ and $i \leq k-m_{r+1}-1$, then $w^{(k-i+1)}=1 w^{(k-i)}$. 


\section{Theorem 4.4.}

$$
c_{u v}^{w}=\# \mathscr{L}_{w / u, v} .
$$

Proof. It follows from (3.1') that

$$
s_{u} s_{v}=\sum_{x} c_{u, 1^{m_{r+1}}}^{x} s_{1^{m} 12 \ldots 1^{m_{r}} 2 x} .
$$

And, by definition, we have

$$
\# \mathscr{L}_{w / u, v}= \begin{cases}\# \mathscr{L}_{x / u, 1^{m_{r+1}}} & \text { if } w=1^{m_{1}} 2 \ldots 1^{m_{r}} 2 x \\ 0 & \text { otherwise. }\end{cases}
$$

Hence it suffices to show the claim in the case where $v=1^{m}$. Now we proceed by induction on $m$. If $m=0$ or 1 , then it is easy to see that

$$
\begin{aligned}
& c_{u, \varnothing}^{w}=\# \mathscr{L}_{w / u, \varnothing}=\delta_{u, w}, \\
& c_{u, 1}^{w}=\# \mathscr{L}_{w / u, 1}= \begin{cases}1 & \text { if } w \triangleright u, \\
0 & \text { otherwise. }\end{cases}
\end{aligned}
$$

If $m \geq 1$, then we have, from Proposition 3.3,

$$
\begin{aligned}
s_{u^{m+1}} s_{1^{m+1}} & =s_{u}\left(s_{1^{m}} s_{1}-s_{1^{m-1}}\right) \\
& =\sum_{y} c_{u, 1^{m}}^{y} s_{y} s_{1}-\sum_{z} c_{u, 1^{m-1}}^{z} s_{z} s_{2} \\
& =\sum_{w}\left(\sum_{y \triangleleft w} c_{u, 1^{m}}^{y}\right) s_{w}-\sum_{z} c_{u, 1^{m-1}}^{z} s_{2 z} .
\end{aligned}
$$

Hence we have

$$
c_{u, 1^{m+1}}^{w}= \begin{cases}c_{u, 1^{m}}^{w^{\prime}} & \text { if } w=1 w^{\prime}, \\ \sum_{y \triangleright w^{\prime \prime}} c_{u, 1^{m}}^{y}-c_{u, 1^{m-1}}^{w^{\prime \prime}} & \text { if } w=2 w^{\prime \prime} .\end{cases}
$$

On the other hand, $\mathscr{L}_{1 w^{\prime} / u, 1^{m+1}}$ consists of the sequences $\left(w^{(0)}, \ldots, w^{(m)}, 1 w^{\prime}\right)$ such that $\left(w^{(0)}, \ldots, w^{(m)}\right) \in \mathscr{L}_{w^{\prime} / u, 1^{m}}$ and $\mathscr{L}_{2 w^{\prime \prime} / u, 1^{m+1}}$ consists of the sequences $\left(w^{(0)}, \ldots, w^{(m)}, 2 w^{\prime \prime}\right)$ such that $\left(w^{(0)}, \ldots, w^{(m)}\right) \in \mathscr{L}_{y / u, 1^{m}}$ for some $y \triangleright w^{\prime \prime}$ and that $\left(w^{(m-1)}, w^{(m)}, 2 w^{\prime \prime}\right)$ is not of the form $\left(w^{\prime \prime}, 1 w^{\prime \prime}, 2 w^{\prime \prime}\right)$. Therefore we obtain the same recurrence:

$$
\# \mathscr{L}_{w / u, 1^{m+1}}= \begin{cases}\# \mathscr{L}_{w^{\prime} / u, 1^{m}} & \text { if } w=1 w^{\prime} \\ \sum_{y \triangleright w^{\prime \prime}} \# \mathscr{L}_{y / u, 1^{m}}-\mathscr{L}_{w^{\prime \prime} / u, 1^{m-1}} & \text { if } w=2 w^{\prime \prime}\end{cases}
$$

So we have $c_{u v}^{w}=\# \mathscr{L}_{w / u, v}$.

\section{YF-ANALOGUE OF POWER SUM SYMMETRIC FUNCTIONS}

Definition. For $v=1^{m_{1}} 21^{m_{2}} \ldots 1^{m_{r}} 21^{m_{r+1}}$, we define

$$
p_{v}=p_{21^{m_{r+1}}} p_{21^{m_{r}}} \ldots p_{21^{m_{2}}} p_{1^{m_{1}}}
$$

where

$$
p_{1^{k}}=s_{1}^{k}, \quad p_{21^{k}}=s_{1}^{k}\left(s_{1}^{2}-(k+2) s_{2}\right) .
$$

We remark that

$$
p_{1 v}=p_{v} p_{1}, \quad p_{2 v}=p_{v}\left(s_{1}^{2}-(m(v)+2) s_{2}\right),
$$


where $m(v)$ is the number of 1 's at the head of $v$. Let $T=\left(T_{v w}\right)$ be the transition matrix from $p$ to $h$ :

$$
p_{v}=\sum_{w} T_{v w} h_{w} .
$$

Then $T$ is the diagonal sum of matrices $T_{n}=\left(T_{v w}\right)_{v, w \in \mathbb{Y F}_{n}}$. We use (5.1) to obtain the following recurrences for $T_{v w}$.

\section{Proposition 5.1.}

(1) $T_{1 v, 1 w}=T_{v w}$.

(2) $T_{1 v, 2 w}=0$.

(3) $T_{2 v, 12 w}=0$.

(4) $T_{2 v, 11 w}=T_{v w}$.

(5) $T_{2 v, 2 w}=-(m(w)+2) T_{v, w}$.

Hence, if $T_{v w} \neq 0$, then $w$ is a refinement of $v$, i.e., $w$ is obtained by replacing some 2 's in $v$ by 11 . In particular, $T_{n}$ is a triangular matrix with respect to the dominance order.

Let $V_{n}=\left(V_{u v}\right)_{u \in \mathrm{YF}_{n-1}, v \in \mathrm{YF}_{n}}$ be the $F_{n-1} \times F_{n}$ matrix defined by

$$
V_{u v}= \begin{cases}1 & \text { if } v=1 u, \\ 0 & \text { otherwise. }\end{cases}
$$

That is, $V_{n}$ is of the form

$$
V_{n}=\left(\begin{array}{ll}
0 & I_{F_{n-1}}
\end{array}\right) .
$$

And let $M_{n}$ be the diagonal matrix whose $(v, v)$-entry is $m(v)$. Then we have

$$
M_{n+1}=\left(\begin{array}{cc}
0 & 0 \\
0 & M_{n}+I
\end{array}\right) \text {. }
$$

Also we can rewrite Proposition 5.1 in matrix form:

$$
T_{n+1}=\left(\begin{array}{cc}
-\left(M_{n-1}+2 I\right) T_{n-1} & T_{n-1} V_{n-1} \\
0 & T_{n}
\end{array}\right) .
$$

Let $X=\left(\chi_{w}^{v}\right)_{w, v \in \mathbb{Y}}$ be the transition matrix from $p$ to $s$ :

$$
p_{w}=\sum_{v} \chi_{w}^{v} s_{v}
$$

Then $X$ is the diagonal sum of matrices $X_{n}=\left(\chi_{w}^{v}\right)_{w, v \in \mathbb{Y F}_{n}}$ and $X_{n}$ is given by

$$
X_{n}=T_{n}{ }^{t} K_{n}
$$

\section{Proposition 5.2.}

$$
\begin{gathered}
X_{n+1}=\left(\begin{array}{cc}
-X_{n-1} & X_{n-1} D_{n} \\
X_{n}^{t} D_{n} & X_{n}
\end{array}\right), \\
X_{n-1} D_{n}=V_{n-1} X_{n} \\
X_{n}{ }^{t} D_{n}={ }^{t} V_{n-1}\left(M_{n-1}+I\right) X_{n-1} .
\end{gathered}
$$


Proof. First we note that

$$
\begin{gathered}
V_{n-1}{ }^{t} K_{n}={ }^{t} K_{n-1} D_{n}, \\
{ }^{t} V_{n-1}\left(M_{n-1}+I\right) V_{n-1}=M_{n} .
\end{gathered}
$$

These are clear from (4.2) and (5.2)-(5.4).

We will prove by induction on $n$. From (4.2) and (5.4), we have

$$
X_{n+1}=\left(\begin{array}{cc}
-\left(M_{n-1}+2 I\right) T_{n-1}{ }^{t} K_{n-1}+T_{n-1} V_{n-1}{ }^{t} K_{n}{ }^{t} D_{n} & T_{n-1} V_{n-1}{ }^{t} K_{n} \\
T_{n}{ }^{t} K_{n}{ }^{t} D_{n} & T_{n}{ }^{t} K_{n}
\end{array}\right) .
$$

Using (5.9) and the induction hypothesis ((5.7) and (5.8)), we see

$$
\begin{aligned}
T_{n-1} V_{n-1}{ }^{t} K_{n}{ }^{t} D_{n} & =X_{n-1} D_{n}{ }^{t} D_{n}=V_{n-1}{ }^{t} V_{n-1}\left(M_{n-1}+I\right) X_{n-1}, \\
T_{n-1} V_{n-1}{ }^{t} K_{n} & =X_{n-1} D_{n} .
\end{aligned}
$$

Hence we obtain (5.6). The relations (5.7) and (5.8) can be shown by matrix computation.

For example,

$$
\left.X_{5}=\begin{array}{lcccccccc}
221 & 212 & 2111 & 122 & 1211 & 1121 & 1112 & 11111 \\
221 & 212 \\
2111 & 1 & -1 & -1 & 0 & 0 & -1 & 1 & 1 \\
1211 & 0 & 1 & -1 & -1 & 1 & 0 & -1 & 1 \\
1121 & 0 & -1 & -1 & 3 & 3 & 2 & 1 & 1 \\
1112 & 0 & 0 & 0 & 1 & -1 & 0 & -1 & 1 \\
11111 & 0 & 1 & 1 & 0 & 0 & -1 & 1 & 1 \\
8 & 4 & 4 & 3 & 3 & 2 & 1 & 1
\end{array}\right) .
$$

We can rewrite (5.6) into the recurrence relations:

$$
\chi_{2 w}^{2 v}=-\chi_{w}^{v}, \quad \chi_{2 w}^{1 v}=\sum_{u \triangleleft v} \chi_{w}^{u}, \quad \chi_{1 w}^{2 v}=\sum_{z \triangleright v} \chi_{w}^{z}, \quad \chi_{1 w}^{1 v}=\chi_{w}^{v} .
$$

By using the induction and these recurrence relations, we see that, for $v, w \in$ $\mathbb{Y F}_{n}$,

$$
\begin{aligned}
\chi_{v}^{1^{n}} & =1, \quad \chi_{1^{n}}^{v}=e(v), \\
\chi_{v}^{1^{n-2} 2} & = \begin{cases}1 & \text { if } v \text { ends with 1, } \\
-1 & \text { if } v \text { ends with 2, }\end{cases} \\
\chi_{v}^{\omega(w)} & =\varepsilon(v) \chi_{v}^{w},
\end{aligned}
$$

where $\varepsilon(v)=\chi_{v}^{1^{n-2} 2}$. Here $\omega$ is a poset automorphism of $\mathbb{Y F}$ defined at the end of Section 3. From the last equation we have $\widetilde{\omega}\left(p_{v}\right)=\varepsilon(v) p_{v}$.

For $v=1^{m_{1}} 21^{m_{2}} 2 \ldots 21^{m_{r+1}} \in \mathbb{Y} \mathbb{F}$, we define

$$
z(v)=m_{1} !\left(m_{2}+2\right) m_{2} ! \ldots\left(m_{r+1}+2\right) m_{r+1} ! .
$$

Then $|v| ! / z(v) \in \mathbb{Z}$ and $\sum_{v \in \mathbb{Y F}_{n}} n ! / z(v)=n !$. Let $Z_{n}$ be the diagonal matrix whose $(v, v)$-entry is $z(v)$. Then we have

$$
Z_{n+1}=\left(\begin{array}{cc}
\left(M_{n-1}+2 I\right) Z_{n-1} & 0 \\
0 & \left(M_{n}+I\right) Z_{n}
\end{array}\right) .
$$




\section{Proposition 5.3.}

$$
X_{n}{ }^{t} X_{n}=Z_{n}
$$

Therefore we have

$$
\left\langle p_{v}, p_{w}\right\rangle=\delta_{v w} z(v) .
$$

Proof. Induction on $n$. By (5.6), we have

$$
X_{n+1}{ }^{t} X_{n+1}=\left(\begin{array}{cc}
X_{n-1}{ }^{t} X_{n-1}+X_{n-1} D_{n}{ }^{t} D_{n}{ }^{t} X_{n-1} & 0 \\
0 & X_{n}{ }^{t} X_{n}+X_{n}{ }^{t} D_{n} D_{n}{ }^{t} X_{n}
\end{array}\right) .
$$

Here we use (5.7), (5.8), and (5.10) to obtain

$$
X_{n+1}{ }^{t} X_{n+1}=\left(\begin{array}{cc}
\left(M_{n-1}+2 I\right) Z_{n-1} & 0 \\
0 & \left(M_{n}+I\right) Z_{n}
\end{array}\right)=Z_{n+1} .
$$

Rewriting (5.7) and (5.8) in terms of $p_{v}$, we obtain the following proposition.

\section{Proposition 5.4.}

$$
U p_{v}=p_{1 v}, \quad D p_{1 v}=m(1 v) p_{v}, \quad D p_{2 v}=0 .
$$

In particular, for any $v \in Y F_{n}, p_{v}$ is an eigenvector for $\left.U D\right|_{R_{n}}: R_{n} \rightarrow R_{n}$ belonging to the eigenvalue $m(v)$. The $p_{v}$ 's give a complete set of orthogonal eigenvectors for $\left.U D\right|_{R_{n}}$.

In the case of Young's lattice or the ring of symmetric functions, the transition matrix $M(p, h)$ (resp. $M(h, s)$ ) from the power sum symmetric functions to the complete symmetric functions (resp. from the complete symmetric functions to the Schur functions) is a triangular matrix under a suitable ordering (dominance order) of rows and columns. And the character table of the symmetric groups is given by $M(p, s)$, the transition matrix from power sum symmetric functions to the Schur functions. Then Proposition 5.3 corresponds to the orthogonality relations for characters. Proposition 5.4 is a $\mathbb{Y F}$-analogue of [S1, Proposition 4.7].

As is shown in [O], each homogeneous component $R_{n}$ admits a structure of associative commutative algebra satisfying the following properties:

(1) If we denote by $*$ the product in $R_{n}$, then $s_{u} * s_{v}=\sum_{w \in \mathrm{YF}_{n}} g_{u v}^{w} s_{w}$ with nonnegative integers $g_{u v}^{w}$.

(2) $S_{1^{n}}$ is the identity element of $R_{n}$.

(3) $R_{n} \otimes_{\mathbb{Z}} \mathbb{Q}$ is a semisimple algebra with minimal idempotents $\frac{1}{z(v)} p_{v}(v \in$ $\left.\mathbb{Y F}_{n}\right)$.

This algebra structure on $R_{n}$ gives an example of fusion algebra at algebraic level. The notion of fusion algebra is a generalization of the character ring of a finite group. (See [B] for fusion algebras at algebraic level.)

\section{Algebras associated to $\mathbb{Y F}^{(r)}$}

Finally we consider the $r$-Young-Fibonacci lattice $\mathbb{Y F}^{(r)}$. Let $K_{0}$ be a field of characteristic 0 such that $K_{0}$ contains a primitive $r$ th root $\zeta$ of unity. We will work with the base field $K=K_{0}\left(x_{i, k}, y_{i}: i=1,2, \ldots, k=0,1, \ldots, r-1\right)$. 
Let $\mathscr{F}_{n}^{(r)}$ be the $K$-algebra defined by the following presentation:

generators : $E_{1}, \ldots, E_{n-1}, t_{1}, \ldots, t_{n}$,

relations : $E_{i} t_{i}^{k} E_{i}=x_{i, k} E_{i} \quad(i=1, \ldots, n-1, k=0, \ldots, r-1)$,

$$
\begin{aligned}
& E_{i} E_{j}=E_{j} E_{i} \quad(\text { if }|i-j| \geq 2), \\
& E_{i+1} E_{i} E_{i+1}=y_{i} E_{i+1} \quad(i=1, \ldots, n-2), \\
& E_{i} t_{i+1}=t_{i+1} E_{i}=E_{i} \quad(i=1, \ldots, n-2), \\
& E_{i} t_{j}=t_{j} E_{i} \quad(j \neq i, i+1), \\
& t_{i}^{r}=1 \quad(i=1, \ldots, n), \\
& t_{i} t_{j}=t_{j} t_{i} \quad(i, j=1, \ldots, n) .
\end{aligned}
$$

We will construct irreducible representations of $\mathscr{F}_{n}^{(r)}$ on the $K$-vector space $V_{v}^{(r)}$ with basis $\Omega^{v}\left(v \in \mathbb{Y F}_{n}^{(r)}\right)$. Define endomorphisms $\pi_{v}^{(r)}\left(E_{i}\right)$ and $\pi_{v}^{(r)}\left(t_{i}\right)$ on $V_{v}^{(r)}$ by putting, for a basis element $T=\left(v^{(0)}, \ldots, v^{(n)}\right) \in \Omega^{v}$,

$$
\begin{aligned}
& \pi_{v}^{(r)}\left(E_{i}\right)\left(v^{(0)}, \ldots, v^{(i-1)}, v^{(i)}, v^{(i+1)}, \ldots, v^{(n)}\right) \\
& = \begin{cases}\sum_{w \triangleright v^{(i-1)}} \frac{\alpha^{(r)}(w)}{\alpha^{(r)}\left(v^{(i-1)}\right)}\left(v^{(0)}, \ldots, v^{(i-1)}, w, v^{(i+1)}, \ldots, v^{(n)}\right) \\
0 \text { otherwise, }\end{cases} \\
& \pi_{v}^{(r)}\left(t_{i}\right)\left(v^{(0)}, \ldots, v^{(i-1)}, v^{(i)}, \ldots, v^{(n)}\right) \\
& = \begin{cases}\zeta^{k}\left(v^{(0)}, \ldots, v^{(i-1)}, v^{(i)}, \ldots, v^{(n)}\right) & \text { if } v^{(i)}=1_{k} v^{(i-1)}, \\
\left(v^{(0)}, \ldots, v^{(i-1)}, v^{(i)}, \ldots, v^{(n)}\right) & \text { otherwise. }\end{cases}
\end{aligned}
$$

Here the coefficients $\alpha^{(r)}(v)\left(v \in \mathbb{Y F}^{(r)}\right)$ are defined as follows: First we introduce a family of polynomials $P_{l}^{k_{1}, \ldots, k_{l}}$ by the following recurrence:

$$
P_{0}=1, \quad P_{1}^{k}=\alpha_{1, k}, \quad P_{l}^{k_{1}, \ldots, k_{l}}=\alpha_{l, k_{1}} P_{l-1}^{k_{2}, \ldots, k_{l}}-\delta_{k_{1}, 0} y_{1} P_{l-2}^{k_{3}, \ldots, k_{l}},
$$

where $\alpha_{l, j}=\frac{1}{r} \sum_{k=0}^{r-1} \zeta^{j k} x_{l, k}$. Then $\alpha^{(r)}(v)$ is defined by

$$
\begin{aligned}
\alpha^{(r)}\left(1_{k_{1}} \ldots 1_{k_{l}}\right) & =P_{l}^{k_{1}, \ldots, k_{l}}, \\
\alpha^{(r)}\left(1_{k_{1}} \ldots 1_{k_{l}} 2\right) & =P_{l+1}^{k_{1}, \ldots, k_{l}, 0}\left[\begin{array}{cc}
x_{1, k} \rightarrow \delta_{k 0} y_{1}, & x_{i, k} \rightarrow x_{i+1, k}(i \geq 2) \\
y_{1} \rightarrow x_{1,0} y_{2}, & y_{i} \rightarrow y_{i+1}(i \geq 2)
\end{array}\right] .
\end{aligned}
$$

In general, for $u \in \mathbb{Y F}_{m}$,

$$
\alpha^{(r)}\left(1_{k_{1}} \ldots 1_{k_{l}} 2 u\right)=\alpha^{(r)}\left(1_{k_{1}} \ldots 1_{k_{l}}\right)\left[x_{i, k} \rightarrow x_{m+i, k}, y_{i} \rightarrow y_{m+i}\right] \alpha(u) .
$$

Then we can check that $\pi_{v}^{(r)}\left(E_{i}\right)$ 's and $\pi_{v}^{(r)}\left(t_{i}\right)$ 's satisfy the fundamental relations of $\mathscr{F}_{n}^{(r)}$. Hence we obtain a representation $\pi_{v}^{(r)}$ of $\mathscr{F}_{n}^{(r)}$ on $V_{v}^{(r)}$.

Theorem 6.1. (1) The algebra $\mathscr{F}_{n}^{(r)}$ is semisimple and of dimension $r^{n} n$ !.

(2) The $V_{v}^{(r)}$ 's $\left(v \in \mathbb{Y F}_{n}^{(r)}\right)$ give a complete set of irreducible $\mathscr{F}_{n}^{(r)}$-modules.

In the same way as in Section 3, we can define a product on $R^{(r)}=\bigoplus_{n \geq 0} R_{n}^{(r)}$, where $R_{r}^{(r)}$ is the free $\mathbb{Z}$-module with basis $\left\{s_{v}: v \in \mathbb{Y F}_{n}^{(r)}\right\}$, and make $R^{(r)}$ an associative graded algebra. 


\section{Proposition 6.2.}

(1) $s_{v} s_{1_{0}}=s_{1_{0} v}+\sum_{w \triangleleft v} s_{2 w}$.

(2) $s_{v} s_{1_{k}}=s_{1_{k} v}$ if $k \neq 0$.

(3) $s_{v} s_{2}=s_{2 v}$.

Theorem 6.3. Let $\mathbb{Z}\left\langle X_{0}, \ldots, X_{r-1}, Y\right\rangle$ be the noncommutative polynomial ring with grading given by $\operatorname{deg} X_{k}=1$ and $\operatorname{deg} Y=2$. Then there exists an algebra isomorphism $\varphi: \mathbb{Z}\left\langle X_{0}, \ldots, X_{r-1}, Y\right\rangle \rightarrow R^{(r)}$ such that $\varphi\left(X_{k}\right)=s_{1_{k}}(k=$ $0,1, \ldots, r-1)$ and $\varphi(Y)=s_{2}$.

Put $R_{\mathbb{C}}^{(r)}=R^{(r)} \otimes_{\mathbb{Z}} \mathbb{C}$ and extend the scalar product $\langle, \quad\rangle$ on $R^{(r)}$ to the Hermitian form $\langle$,$\rangle on R_{\mathbb{C}}^{(r)}$. A correspondent to the power sum symmetric functions is defined as follows:

$$
\begin{gathered}
p_{\varnothing}=1, \quad p_{1_{k}}=\sum_{j=0}^{r-1} \zeta^{j k} s_{1_{j}}, \\
p_{1_{k} v}=p_{v} p_{1_{k}}, \quad p_{2 v}=p_{v}\left(p_{1_{0}}^{2}-r\left(m^{0}(v)+2\right) s_{2}\right),
\end{gathered}
$$

where $m^{0}(v)$ is the number of $1_{0}$ 's at the head of $v$. And we define $z^{(r)}(v)$ $\left(v \in \mathbb{Y F}^{(r)}\right)$ by the following recurrence:

$$
\begin{aligned}
z^{(r)}(\varnothing) & =1, \\
z^{(r)}\left(1_{k} v\right) & = \begin{cases}r\left(m^{0}(v)+1\right) z^{(r)}(v) & \text { if } k=0, \\
r z^{(r)}(v) & \text { if } k \neq 0,\end{cases} \\
z^{(r)}(2 v) & =r^{2}\left(m^{0}(v)+2\right) z^{(r)}(v) .
\end{aligned}
$$

Then we have

Proposition 6.4. For $v, w \in \mathbb{Y F}^{(r)}$, we have

$$
\left\langle p_{v}, p_{w}\right\rangle=\delta_{v w} z^{(r)}(v) .
$$

Moreover we have

$$
U p_{v}=p_{1_{0} v}, \quad D p_{1_{k} v}=\delta_{k 0} r m^{0}\left(1_{0} v\right) p_{v}, \quad D p_{2 v}=0 .
$$

In particular, for any $v \in \mathbb{Y F}_{n}^{(r)}, p_{v}$ is an eigenvector for $\left.U D\right|_{R_{n}^{(r)}}: R_{n}^{(r)} \rightarrow$ $R_{n}^{(r)}$ belonging to the eigenvalue $m^{0}(v)$. And the $p_{v}$ 's give a complete set of orthogonal eigenvectors for $\left.U D\right|_{R_{n}^{(r)}}$.

\section{REFERENCES}

[B] E. Bannai, Association schemes and fusion algebras (an introduction), J. Algebraic Combin. 2 (1993), 327-344.

[F1] S. V. Fomin, Generalized Robinson-Schensted-Knuth correspondence, J. Soviet Math. 41 (1988), 979-991.

[F2] — Duality of graded graphs, Report No.15 (1991/92), Institut Mittag-Leffler.

[F3] - Schensted-type algorithms for dual graded graphs, Report No.16 (1991/92), Institut Mittag-Leffler.

[GHJ] F. M. Goodman, P. de la Harpe, and V. F. R. Jones, Coxeter graphs and towers of algebras, Math. Sci. Res. Inst. Publ., no. 14, Springer-Verlag, New York, 1989. 
[HR] T. Halverson and A. Ram, Character of algebras containing a Jones basic construction: the Temperley-Lieb, Okada, Brauer, and Birman-Wenzl algebras, preprint.

[JK] G. James and A. Kerber, The representation theory of the symmetric groups, Encyclopedia of Mathematics and Its Applications 16, Addison-Wesley, Reading, MA, 1981.

[KM] M. Kosuda and J. Murakami, Centralizer algebras of the mixed tensor representations of quantum group $U_{q}(\mathfrak{g l}(n, \mathbb{C}))$, Osaka J. Math. 30 (1993), 475-507.

[M] I. G. Macdonald, Symmetric functions and Hall polynomials, Oxford Univ. Press, Oxford, 1979.

[O] S. Okada, Reflection-extension of fusion algebras, preprint.

[R1] T. W. Roby, Applications and extensions of Fomin's generalization of the Robinson-Schensted correspondence to differential posets, Ph.D. thesis, Massachusetts Institute of Technology, 1991.

[R2] Schensted correspondences for differential posets, preprint.

[S1] R. P. Stanley, Differential posets, J. Amer. Math. Soc. 1 (1988), 919-961.

[S2] _ Variations on differential posets, Invariant Theory and Tableaux (D. Stanton, ed.), IMA Vol. Math. Appl., no. 19, Springer, New York, 1988, pp. 145-165.

[S3] - Further combinatorial properties of two Fibonacci lattices, European. J. Combin. 11 (1990), 181-188.

[W] H. Wenzl, Hecke algebras of type $A_{n}$ and subfactors, Invent. Math. 92 (1988), 349-383.

Department of Mathematics, Nagoya University, Chikusa-ku, Nagoya 464-01, Japan

E-mail address: okada@math.nagoya-u.ac.jp 